\title{
Active Control of Contact Force for a Pantograph-Catenary System
}

\author{
Jiqiang Wang \\ Jiangsu Province Key Laboratory of Aerospace Power Systems, College of Energy and Power Engineering, \\ Nanjing University of Aeronautics and Astronautics, Nanjing 210016, China
}

Correspondence should be addressed to Jiqiang Wang; jiqiang_wang@hotmail.com

Received 18 November 2015; Accepted 20 December 2015

Academic Editor: Mickaël Lallart

Copyright (C) 2016 Jiqiang Wang. This is an open access article distributed under the Creative Commons Attribution License, which permits unrestricted use, distribution, and reproduction in any medium, provided the original work is properly cited.

The performance of the high speed trains depends critically on the quality of the contact in the pantograph-catenary interaction. Maintaining a constant contact force needs taking special measures and one of the methods is to utilize active control to optimize the contact force. A number of active control methods have been proposed in the past decade. However, the primary objective of these methods has been to reduce the variation of the contact force in the pantograph-catenary system, ignoring the effects of locomotive vibrations on pantograph-catenary dynamics. Motivated by the problems in active control of vibration in large scale structures, the author has developed a geometric framework specifically targeting the remote vibration suppression problem based only on local control action. It is the intention of the paper to demonstrate its potential in the active control of the pantograph-catenary interaction, aiming to minimize the variation of the contact force while simultaneously suppressing the vibration disturbance from the train. A numerical study is provided through the application to a simplified pantograph-catenary model.

\section{Introduction}

The quality of the contact between pantograph and catenary has played an essential role in delivering electrical energy to high speed trains. However the contact force varies strongly with speed and over spans, causing excessive wears or even lost contact. Measures have to be taken to maintain a relatively constant contact force in the pantographcatenary interaction. A traditional pantograph system is passive consisting of viscous-elastic components with fixed parameters, designed for fixed type of catenaries and locomotive velocities. This obviously prevents its utilization in modern high speed trains such as ICE, TGV or CRH. One method to achieve the uniformity of the contact force is to make use of advanced materials for pantograph slide plate (see [1] for a review on recent development), while another is to take advantage of the development in control theory-this is the concept of active control of pantographcatenary system in order to maintain an optimal contact force. Indeed this has attracted much attention in the study of pantograph-catenary interaction. For example, simple state feedback control strategy is considered in $[2,3]$; in [4], an LQR is designed using a linear pantograph-catenary model, while in [5] a high order sliding mode variable structure controller is constructed for the active control of pantograph; an evolutionary multiobjective optimization approach is utilized taking into account the perturbations caused by the time-varying stiffness of catenary [6]; differential-geometric theory is used for output-perturbation decoupling for a nonlinear pantograph-catenary model in [7]; very recently fuzzy logic has also found application in the active control strategies such as in $[8,9]$.

In the above papers, the primary objective of active control has been to reduce the variation of the contact force in the pantograph-catenary system, while it is also well-known that the locomotive vibrations on pantographcatenary dynamics are nonnegligible and the effect becomes particularly violent with the increasing speed of the train $[10$, 11]. As a consequence, it is essential to attenuate the vibration disturbance while minimizing the variation of the contact force. In principle this can be achieved through decoupling control with each controller dealing with contact force and vibration disturbance separately, but implementation cost increases with system complexity. In this paper, a geometric 
design framework is introduced, aiming to minimize the variation of the contact force while simultaneously suppressing the vibration disturbance from the train. The paper is structured as follows: Section 2 describes the pantograph-catenary model; Section 3 briefly reviews the design procedures and presents the simulation results; finally Section 4 concludes the paper and comments the further development.

\section{Simplified Pantograph-Catenary Model}

2.1. Pantograph Model. A DSA250 pantograph is shown in Figure 1(a), which is used in CRH1, CRH2, and CRH5 EMUs, with a designed speed of $250 \mathrm{~km} / \mathrm{h}$. Some complex pantograph models exist but to be elementary, a pantograph can be modelled as a dual-mass system with known masses representing the head and frame, along with appropriate springs and dampers. A configuration is illustrated in Figure 1(b).

In Figure $1, m_{1}$ and $m_{2}$ are the equivalent mass of the collector head and pantograph frame, with $Z_{1}$ and $Z_{2}$ denoting their displacement relative to the equilibrium; $c_{1}$ and $k_{k}$ are the parameters of the spring-damper structure connecting the collector head and pantograph frame; $c_{2}$ is the viscosity coefficient of the pantograph frame; $F_{0}, f_{k}$, and $f_{c}$ are static lifting force, elastic force, and damping force, respectively; $\dot{Z}_{r}$ represents the vibration disturbance transmitting from the locomotive and finally $u$ is the required force for active control.

2.2. Catenary Model. The suspension catenary in a single span is shown in Figure 2.

Usually the rigidity of the suspension catenary $k(t)$ is a time-varying function affected by the speed of the locomotive, type of the catenaries and its corresponding parameters, and so forth. It can be obtained. One of the methods to obtain an expression of $k(t)$ is through the application of finite element method to practical catenaries leading to

$$
\begin{aligned}
& k(t)=k_{0}\left(1+a_{1} \cos \frac{2 \pi}{L} t+a_{2} \cos \frac{2 \pi}{L_{1}} t\right. \\
& +a_{3}\left(\cos \frac{2 \pi}{L} t\right)^{2}+a_{4}\left(\cos \frac{\pi}{L} t\right)^{2}+a_{5}\left(\cos \frac{\pi}{L_{1}} t\right)^{2} \\
& +\cdots)
\end{aligned}
$$

and the parameters are then estimated by a nonlinear least squares approximation.

2.3. Pantograph-Catenary Interaction Model. For pantograph-catenary interaction, the catenary can be modeled as a spring with time varying rigidity $k(t)$. With the expression for $k(t)$ the contact force $F$ shown in Figure 1 is

$$
F=k(t) Z_{1} \text {. }
$$

Hence combining the pantograph model (Figure 1(b)) with the contact force from suspension catenary, a simplified pantograph-catenary interaction model can be obtained as shown in Figure 3.

Then it is easy to show the above model can be described by the following equations:

$$
\begin{aligned}
m_{1} \ddot{Z}_{1}+k(t) Z_{1}+f_{k}+f_{c} & =u, \\
m_{1} \ddot{Z}_{2}+c_{2}\left(\dot{Z}_{2}-\dot{Z}_{r}\right)-f_{k}-f_{c} & =F_{0}-u, \\
f_{k} & =k_{k}\left(Z_{1}-Z_{2}\right), \\
f_{c} & =c_{1}\left(\dot{Z}_{1}-\dot{Z}_{2}\right) .
\end{aligned}
$$

The objective of active control can then be stated as follows: finding a control action $u$ based only on the displacement $Z_{1}$, such that the variation of both the contact force $F$ including the effects of the disturbance transmission force $\dot{z}_{r}$ is reduced simultaneously.

Rewriting (3) in state space representation is as follows:

$$
\begin{aligned}
& \dot{x}(t)=A x(t)+B \zeta(t), \\
& y(t)=C x(t),
\end{aligned}
$$

where $x^{T}=\left[\begin{array}{llll}Z_{1} & \dot{Z}_{1} & Z_{2} & \dot{Z}_{2}\end{array}\right]^{T}, \zeta^{T}=\left[\begin{array}{ll}u & d\end{array}\right]^{T}$, and $y^{T}=$ $\left[\begin{array}{ll}Z_{1} & Z_{2}\end{array}\right]^{T}$ with $d \equiv F_{0} / c_{2}+\dot{Z}_{r}$; the corresponding matrices are

$$
\begin{aligned}
A & =\left[\begin{array}{cccc}
0 & 1 & 0 & 0 \\
-\frac{k_{k}+k_{0}}{m_{1}} & -\frac{c_{1}}{m_{1}} & \frac{k_{k}}{m_{1}} & \frac{c_{1}}{m_{1}} \\
0 & 0 & 0 & 1 \\
\frac{k_{k}}{m_{2}} & \frac{c_{1}}{m_{2}} & -\frac{k_{k}}{m_{2}} & -\frac{c_{1}+c_{2}}{m_{2}}
\end{array}\right], \\
B & =\left[\begin{array}{cc}
0 & 0 \\
\frac{1}{m_{1}} & 0 \\
0 & 0 \\
-\frac{1}{m_{2}} & \frac{c_{2}}{m_{2}}
\end{array}\right], \\
C & =\left[\begin{array}{cccc}
1 & 0 & 0 & 0 \\
0 & 0 & 1 & 0
\end{array}\right] .
\end{aligned}
$$

The objective of active control presented above can then be restated as finding a local control action using only the information from $Z_{1}$ or

$$
u=-K Z_{1}
$$

such that both $Z_{1}$ and $Z_{2}$ are attenuated simultaneously. 


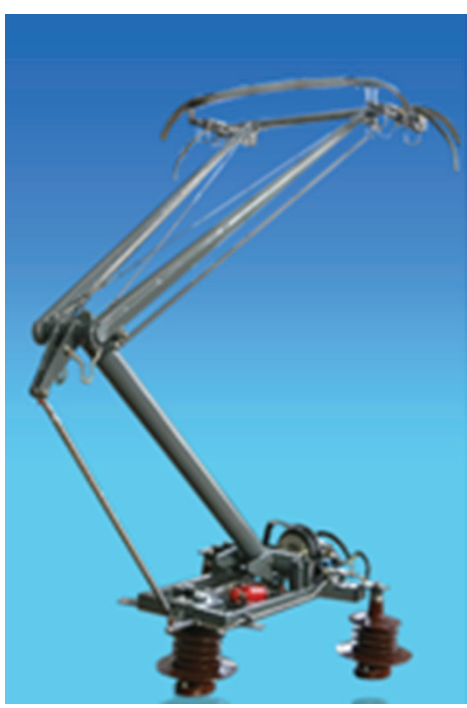

(a)

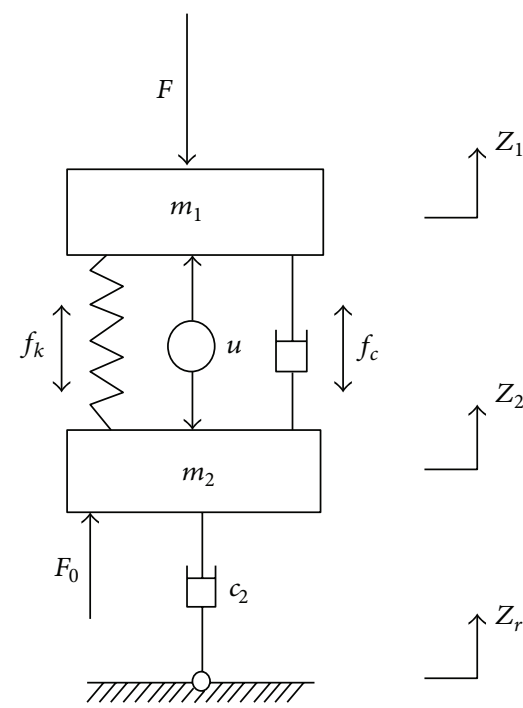

(b)

FIGURE 1: (a) DSA250 produced by CED Railway Electric Tech. Co. (b) A simplified dual-mass pantograph model.

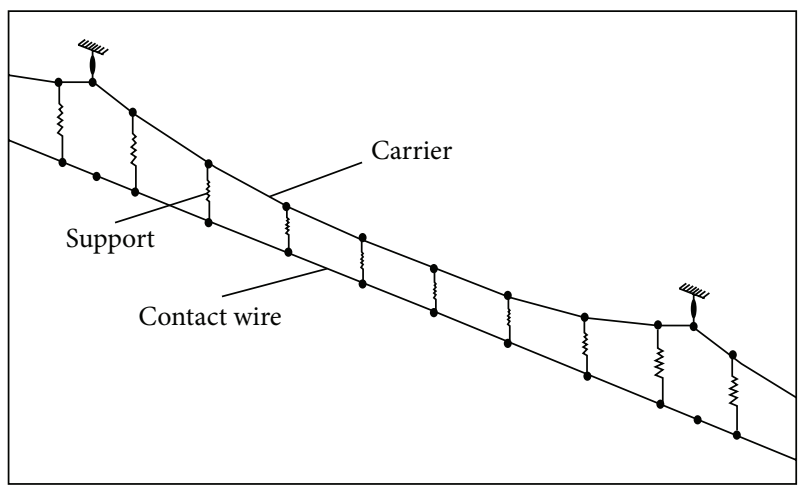

FIgURE 2: The suspension catenary in a single span.

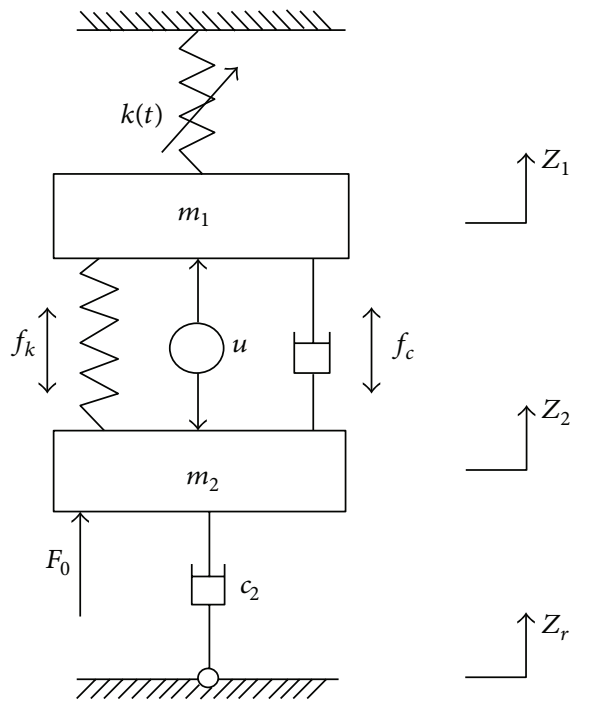

FIGURE 3: A simplified pantograph-catenary interaction model.

\section{Geometric Design for Active Control}

3.1. Geometric Design Procedures. To proceed, first consider the dynamics at a certain frequency $\omega$, and then (4) can be written in the following general form:

$$
\left[\begin{array}{l}
z_{1}(j \omega) \\
z_{2}(j \omega)
\end{array}\right]=\left[\begin{array}{ll}
g_{11}(j \omega) & g_{12}(j \omega) \\
g_{21}(j \omega) & g_{22}(j \omega)
\end{array}\right]\left[\begin{array}{l}
u(j \omega) \\
d(j \omega)
\end{array}\right]
$$

and the required control action has the form:

$$
u(j \omega)=-K(j \omega) z_{1}(j \omega) .
$$

Now denote the sensitivity at a discrete frequency $\omega=\omega_{0}$ by $S\left(j \omega_{0}\right)$ and further define

$$
\begin{aligned}
\alpha\left(j \omega_{0}\right) & =S\left(j \omega_{0}\right)-1 \\
\alpha\left(j \omega_{0}\right) & =\beta\left(j \omega_{0}\right) g\left(j \omega_{0}\right) \\
\text { with } g\left(j \omega_{0}\right) & =\frac{g_{11}\left(j \omega_{0}\right) g_{22}\left(j \omega_{0}\right)}{g_{12}\left(j \omega_{0}\right) g_{21}\left(j \omega_{0}\right)} .
\end{aligned}
$$

Hence the reduction in $z_{1}(j \omega)$ and $z_{2}(j \omega)$ for the discrete frequency $\omega=\omega_{0}$ is equivalent to satisfying the following conditions, respectively:

$$
\begin{aligned}
& |\alpha(j \omega)+1|<1, \\
& |\beta(j \omega)+1|<1 .
\end{aligned}
$$

As (11) relating $\alpha(j \omega)$ to $\beta(j \omega)$ defines a Möbius transformation; then the mapping of (10) on complex $\alpha$-plane is a circle (and its interior) with centre at $-g\left(j \omega_{0}\right)$ and radius $\left|g\left(j \omega_{0}\right)\right|$. Hence it can be concluded that simultaneous reduction of $z_{1}(j \omega)$ and $z_{2}(j \omega)$ is achievable for this discrete frequency $\omega=\omega_{0}$ if and only if the mapping of the unit circle 
(and its interior) $|\beta(j \omega)+1|<1$ on the complex $\alpha$-plane intersects the unit $\alpha$ circle (and its interior) $|\alpha(j \omega)+1|<$ 1. Finally it is noted that an optimal line jointing $(-1,0)$ with $\left(-g\left(j \omega_{0}\right)\right)$ on the complex $\alpha$-plane can be defined and desired levels of attenuation can be obtained by choosing an appropriate point on the line; for example, a choice at point $-g\left(j \omega_{0}\right)$ provides infinite attenuation in $z_{2}\left(j \omega_{0}\right)$. This situation is illustrated in Figure 4.

Based on the development for the discrete frequency $\omega=$ $\omega_{0}$, the aim of reducing $z_{1}(j \omega)$ and $z_{2}(j \omega)$ over an arbitrary frequency band $\left[\omega_{1}, \omega_{N}\right]$ can be approached on a frequencyby-frequency basis and this results in an optimal trajectory on the complex $\alpha$-plane; see Figure 4 . However given the optimal trajectory, a problem arises whether there exists a transfer function $\alpha(s) \in H_{\infty}$ such that $\alpha\left(j \omega_{i}\right)=\alpha_{i} \forall i \in[1, N]$ and $\sup _{s}|\alpha(s)| \leq M$, where $H_{\infty}$ is the Hardy space of bounded analytic functions in the right half plane $\mathfrak{R}(s) \geq 0$ and $M>0$ is a real number.

This is in fact a well-known Nevanlinna-Pick interpolation problem. The problem of the existence of an interpolating transfer function can be answered by the following modified Pick condition.

Modified Pick condition for the existence of a stable interpolating transfer function: there exists a stable transfer function $\alpha(s) \in H_{\infty}$ that interpolates the prescribed trajectory on the complex $\alpha$-plane if and only if the Pick matrix

$$
P=\left[\frac{1-\alpha_{k} \overline{\alpha_{l}} / M^{2}}{j\left(\omega_{k}-\omega_{l}\right)+2 a}\right]_{1 \leq k, l \leq N}
$$

is positive definite.

Remark 1 . The minimal degree of stability $a$ and $M$ can also be considered as design freedom since they can effectively influence the performance outside the frequency band $\left[\omega_{1}, \omega_{N}\right]$.

The positive definiteness of the Pick matrix will ensure the existence of an interpolating transfer function. But the question of how to modify the optimal trajectory in the case of nonpositive definiteness of the Pick matrix remains to be answered: if an optimal solution is not feasible, a suboptimal one will have to be sought to provide the best approximation to the initial optimal choice. The solution comes from using an LMI solver. However the Pick condition must be transformed to an LMI form to use the LMI solver.

Rewrite the Pick matrix as follows:

$$
\begin{aligned}
P= & {\left[\frac{1-\alpha_{k} \overline{\alpha_{l}} / M^{2}}{j\left(\omega_{k}-\omega_{l}\right)+2 a}\right]_{1 \leq k, l \leq N} } \\
= & {\left[\frac{1}{j\left(\omega_{k}-\omega_{l}\right)+2 a}\right]_{1 \leq k, l \leq N} } \\
& -\left[\frac{\left(\alpha_{k} / M\right) \times\left(\overline{\alpha_{l}} / M\right)}{j\left(\omega_{k}-\omega_{l}\right)+2 a}\right]_{1 \leq k, l \leq N} .
\end{aligned}
$$

Define

$$
\begin{aligned}
& T_{0}=\left[\frac{1}{j\left(\omega_{k}-\omega_{l}\right)+2 a}\right]_{1 \leq k, l \leq N}, \\
& Q=\operatorname{blockdiag}\left(\frac{\alpha_{1}}{M}, \ldots, \frac{\alpha_{N}}{M}\right) .
\end{aligned}
$$

We then have

$$
P=T_{0}-Q T_{0} Q^{*} \quad\left({ }^{*} \text { is the Hermitian operator }\right) .
$$

It is known that $T_{0}>0$ hence by applying the Schur complement to (16). We have the following: $P$ is positive definite if and only if

$$
\left[\begin{array}{cc}
T_{0} & Q \\
Q^{*} & T_{0}^{-1}
\end{array}\right]>0 .
$$

The solution to the above LMIs will result in an optimal solution that guarantees a stable interpolating transfer function $\alpha(s)$. The controller $K(s)$ is then obtained:

$$
K(s)=-\frac{\alpha(s)}{[\alpha(s)+1] g_{11}(s)} .
$$

The above design procedures are summarized as follows.

Step 1. Choose control architecture so that $z_{1}-u$ is collocated (a stable $g_{11}$ can facilitate the design but is not an absolute requirement).

Step 2. From the system plant, determine the geometry of $\alpha$ and $\beta$.

Step 3. Depending on the design objective, choose appropriate $\alpha(j \omega)$ trajectory.

Step 4. Find a stable function $\alpha(s)$ such that it interpolates the optimal trajectory $\alpha=S-1$ designed in Step 3.

Step 5. Implement the feedback controller $K$ as in (13).

3.2. Simulation Results. To verify the effectiveness of the design methodology, consider the following numerical values in system dynamics:

$$
\begin{aligned}
& m_{1}=m_{2}=1 \mathrm{~kg} ; \\
& F_{0}=80 \mathrm{~N} ; \\
& c_{2}=30 \mathrm{~N} \cdot \mathrm{s} / \mathrm{m} ; \\
& k_{k}=10, \\
& c_{1}=10 .
\end{aligned}
$$

The rigidity of the suspension catenary is assumed to be

$$
\begin{aligned}
k(t) & =k_{0}\left(1+a_{1} \cos \frac{2 \pi}{L} t+a_{2} \cos \frac{2 \pi}{L_{1}} t\right. \\
+ & \left.a_{3}\left(\cos \frac{2 \pi}{L} t\right)^{2}+a_{4}\left(\cos \frac{\pi}{L} t\right)^{2}\right),
\end{aligned}
$$




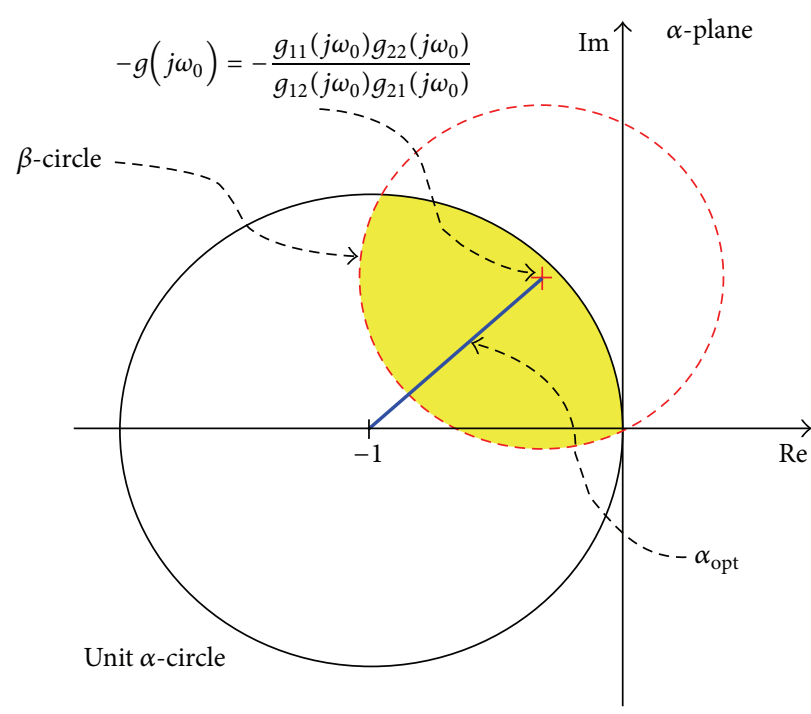

(a)

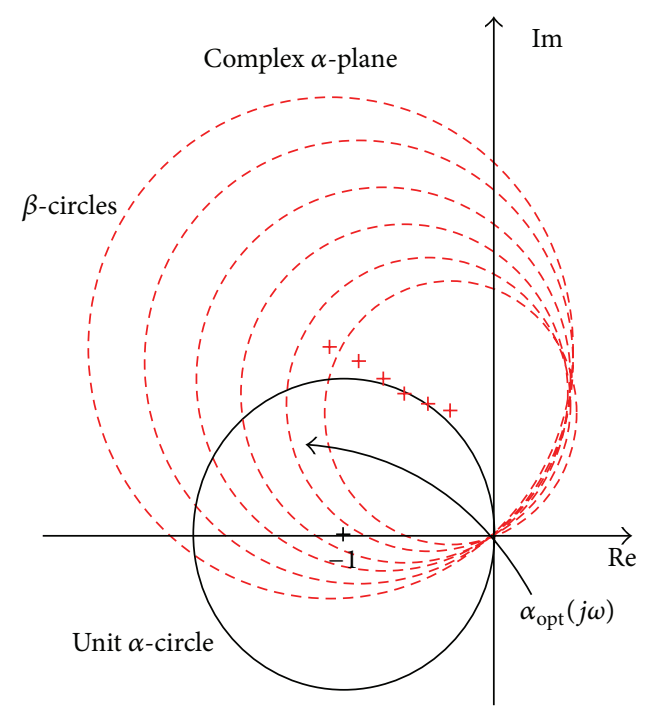

(b)

FIgURE 4: (a) Mapping of $|\beta(j \omega)+1|<1$; (b) optimal solution $\alpha_{\text {opt }}(j \omega)$ on the complex $\alpha$-plane for broad band control.

where $k_{0}=30, L=65 \mathrm{~m}, L_{1}=6 \mathrm{~m}, a_{1}=0.5, a_{2}=0.1$, $a_{3}=0.2$, and $a_{4}=-0.2$

Further assume the scenario of optimal control is to provide optimal reduction in $z_{1}$ (hence the variation of the contact force $F$ is minimized) but without causing vibration enhancement in $z_{2}$ (hence the transmission force from the locomotive to pantograph is attenuated). Then a series of LMIs constraining the values of $\alpha(j \omega)$ within the desired limit of optimal choices is used to obtain the optimal values $\alpha(j \omega)$ over the desired frequency band; an application of the NP algorithm results in a transfer function $\alpha(s)$ with 5 th order that interpolates the optimal $\alpha(j \omega)$ values. The optimal controller $K(s)$ can then be obtained from (18).

With this optimal controller $K(s)$, consider the case that the velocity of the locomotive is $v=200 \mathrm{~m} / \mathrm{s}$; then the performance of the active controller is shown in Figure 5. From Figure 5, it is seen clearly that simultaneous vibration reduction in both $z_{1}(j \omega)$ and $z_{2}(j \omega)$ is achieved using only the feedback of the displacement $Z_{1}$, which is remarkable (almost $6 \mathrm{~dB}$ attenuation is achieved!). The vibration reduction in $Z_{1}$ leads to the reduction of the variation in contact force $F$ :

$$
\begin{aligned}
F= & k(t) Z_{1}=\left[k _ { 0 } \left(1+a_{1} \cos \frac{2 \pi}{L} t+a_{2} \cos \frac{2 \pi}{L_{1}} t\right.\right. \\
& \left.\left.+a_{3}\left(\cos \frac{2 \pi}{L} t\right)^{2}+a_{4}\left(\cos \frac{\pi}{L} t\right)^{2}\right)\right] \times Z_{1} .
\end{aligned}
$$

This is validated in Figure 6.

Now increase the speed of the locomotive to $v=400 \mathrm{~m} / \mathrm{s}$, and the variation of the contact force $F$ is shown in Figure 7, where again the active controller is turned on around 500th second.

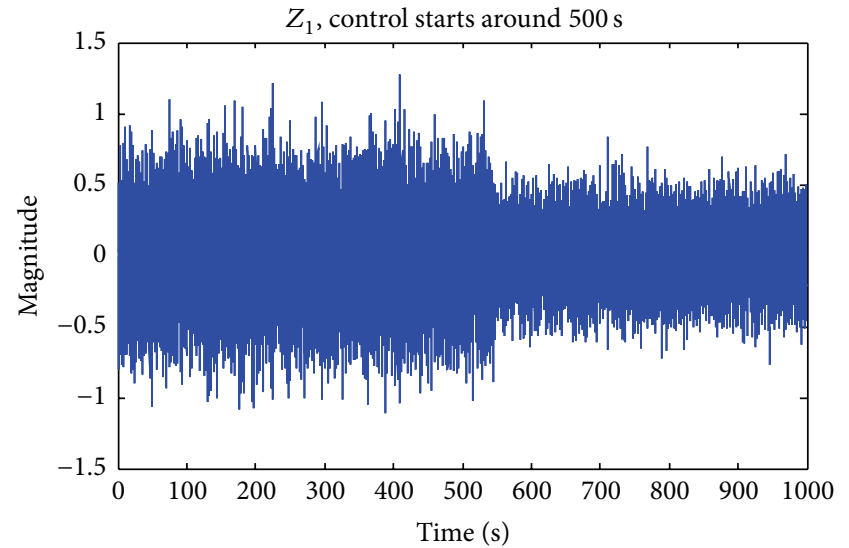

(a)

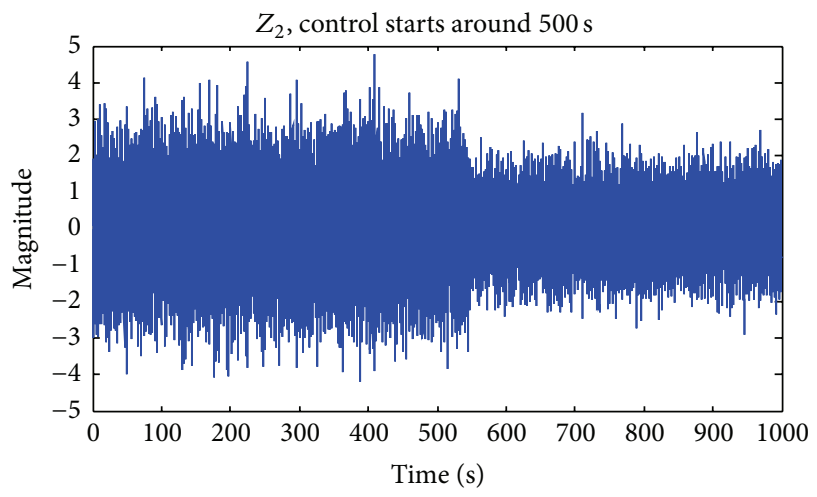

(b)

FIGURE 5: The performance of the active controller: (a) shows the reduction of variation in $z_{1}$ and (b) shows the attenuation of vibration transmission force in $z_{2}$. The controller is turned on around 500th s. 


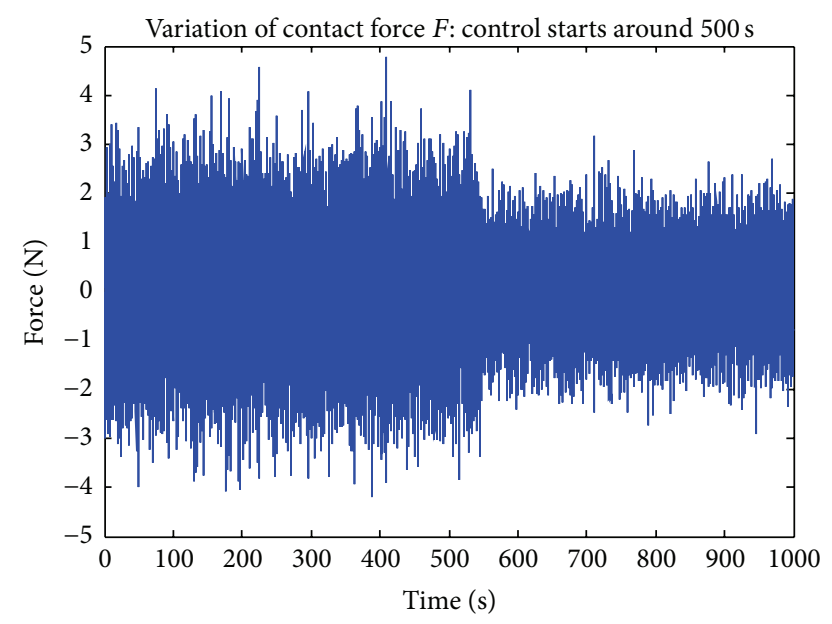

FIGURE 6: The reduction of variation in contact force $F$ for $v=$ $200 \mathrm{~m} / \mathrm{s}$.

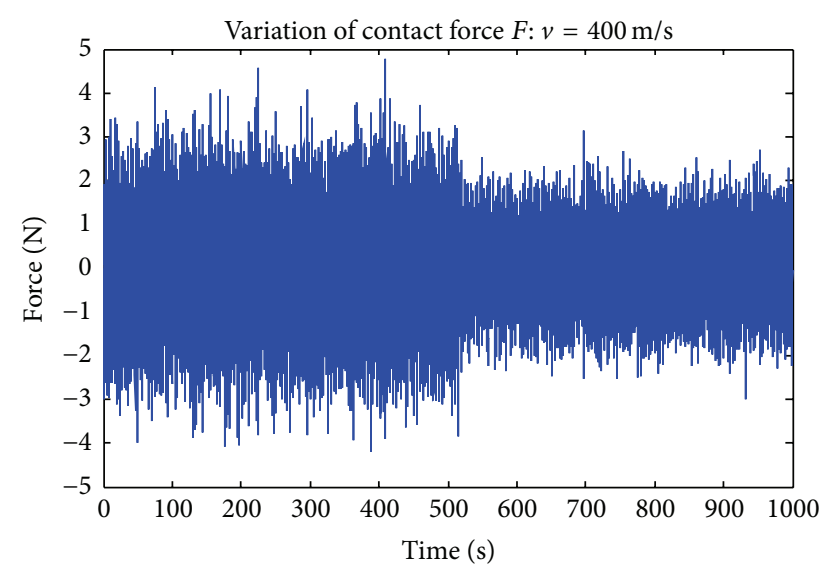

FIGURE 7: The reduction of variation in contact force $F$ for $v=$ $400 \mathrm{~m} / \mathrm{s}$.

It is seen that the same controller is capable of reducing the variation of the contact force over a wide range of the locomotive speed, demonstrating the potential practicality of the above design methodology.

Remark 2. The disturbance in the above simulation is assumed to be a random force with a spectrum over $[2,10] \mathrm{Hz}$ frequency band.

Remark 3. In fact simulation results also conform with the above declaration when the locomotive speed varies with any velocity. This is a strong indication for the effectiveness of the active control strategy in practical applications.

\section{Conclusion}

A simplified model for the pantograph-catenary system has been presented and a geometric design methodology has been introduced for simultaneous variation reduction in both the contact force and the transmission force from the locomotive. The proposed method has been formulated in frequency domain permitting both harmonic and broad band vibration force attenuation. This feature is very desirable since it allows performance shaping at any discrete frequency or over any designated frequency band. Conventional design approaches can only handle this issue through the use of weighting functions, and this is often of rule-of-thumb nature. Indeed, as in the high speed train pantograph-catenary structure considered in the paper, the frequency band can often be estimated for the problematic disturbance forces in many practical industrial systems. And the proposed method thus presents a novel solution to the active control problems. This forms one contribution. Another contribution of the paper is that the proposed strategy is different from the conventional approaches where the objective has been solely to reduce the variation of the contact force in the pantograph-catenary system, without consideration of vibration transmission from the locomotive. Such an active control strategy is novel and deserves further exploration. The simulation results clearly validate the claim that the proposed methodology is very effective in active control of pantograph-catenary interaction.

Further development must utilize a more accurate and realistic model than the one in this note. Indeed, modeling of pantograph-catenary interaction has found much interest and many aspects have been considered; for example, [12] considers a partial differential algebraic equation (a partial differential equation for the catenary and a differential algebraic equation for the pantograph.) for the pantographcatenary system, while both static and dynamic analyses of a catenaries system have been conducted through a high performance computing algorithm in [13]; a finite element model of the catenary system is described with the pantograph system detailed by a multibody model [14]; even the effect of locomotive vibrations on pantograph-catenary system dynamics has been studied in [15], demonstrating the nonnegligibility of locomotive vibrations in high speed operation (as considered in this note); as yet another example, [16] studies the pantograph-catenary interaction considering two independent series of the catenary spans where transition spans are overlapped. Utilization of these models will pave the way of applying the proposed design methodology to the practical pantograph-catenary interaction system.

\section{Conflict of Interests}

The author declares that there is no conflict of interests regarding the publication of this paper.

\section{Acknowledgments}

This work is supported by the Natural Science Foundation of Jiangsu Province (No. BK20140829), Jiangsu Postdoctoral Science Foundation (No. 1401017B), and the Fundamental Research Funds for the Central Universities (No. NS2016024).

\section{References}

[1] J. Xiao, P. Zhang, Y. Du, and H. Liu, "Development of materials for electric locomotive pantograph slide plate," Railway Locomotive \& Car, vol. 25, no. 6, pp. 65-68, 2005 (Chinese). 
[2] T. Wu X and M. J. Brennan, "Active vibration control of a railway pantograph," Proceedings of the IME, Part F: Journal of Rail \& Rapid Transit, vol. 211, no. 2, pp. 117-130, 1997.

[3] G. Poetsch, J. Evans, R. Meisinger et al., "Pantograph/catenary dynamics and control," Vehicle System Dynamics, vol. 28, no. 23, pp. 159-195, 1997.

[4] D. N. O'Connor, S. D. Eppinger, W. P. Seering, and D. N. Wormley, "Active control of a high-speed pantograph," Journal of Dynamic Systems, Measurement and Control, vol. 119, no. 1, pp. 1-4, 1997.

[5] A. Levant, A. Pisano, and E. Usai, "Output-feedback control of the contact-force in high-speed-train pantographs," in Proceedings of the 40th IEEE Conference on Decision and Control (CDC '01), pp. 1831-1836, Orlando, Fla, USA, December 2001.

[6] Y. Lin, C. Lin, and N. Shieh, "Robust optimal design for active pantograph suspension of light rail vehicles," in Proceedings of the 25th IASTED International Conference on Modeling, Identification and Control, pp. 396-401, Lanzarote, Spain, February 2006.

[7] J. Guo, S. Yang, and Q. Zhu, "Research on high speed train pantograph/catenary contact under control with differential geometry theory," Journal of Mechanical Strength, vol. 27, no. 3, pp. 320-323, 2005.

[8] S. Rusu-Anghel, C. Miklos, J. Averseng, and G. O. Tirian, "Control system for catenary-pantograph dynamic interaction force," in Proceedings of the IEEE International Joint Conferences on Computational Cybernetics and Technical Informatics (ICCCCONTI '10), pp. 181-186, Timisoara, Romania, May 2010.

[9] S. Walters, "Simulation of fuzzy control applied to a railway pantograph-catenary system," in Knowledge-Based and Intelligent Information and Engineering Systems, vol. 6277 of Lecture Notes in Computer Science, pp. 322-330, Springer, Berlin, Germany, 2010.

[10] W. Zhang, J. Zeng, and Y. Li, "A review of vehicle system dynamics in the development of high-speed trains in China," International Journal of Dynamics and Control, vol. 1, no. 1, pp. 81-97, 2013.

[11] W. Zhang and S. Zhang, "Dynamics and service simulation for general coupling system of high-speed trains," Journal of Southwest Jiaotong University, vol. 43, no. 2, pp. 147-152, 2008 (Chinese).

[12] M. Arnold and B. Simeon, "The simulation of pantograph and catenary: a PDAE approach," Tech. Rep. 1990, Technische Universität Darmstadt, Fachbereich Mathematik, 1998.

[13] A. Alberto, J. Benet, E. Arias, D. Cebrian, T. Rojo, and F. Cuartero, "A high performance tool for the simulation of the dynamic pantograph-catenary interaction," Mathematics and Computers in Simulation, vol. 79, no. 3, pp. 652-667, 2008.

[14] F. G. Rauter, J. Pombo, J. Ambrósio, and M. Pereira, "Multibody modeling of pantographs for pantograph-catenary interaction," in IUTAM Symposium on Multiscale Problems in Multibody System Contacts, vol. 1 of IUTAM Bookseries, pp. 205-226, Springer, Dordrecht, The Netherlands, 2007.

[15] W. M. Zhai and C. B. Cai, "Effect of locomotive vibrations on pantograph-catenary system dynamics," Vehicle System Dynamics, vol. 29, no. 1, pp. 47-58, 1998.

[16] J. Benet, A. Alberto, E. Arias, and T. Rojo, "A mathematical model of the pantograph-catenary dynamic interaction with several contact wires," IAENG International Journal of Applied Mathematics, vol. 37, no. 2, 2007. 


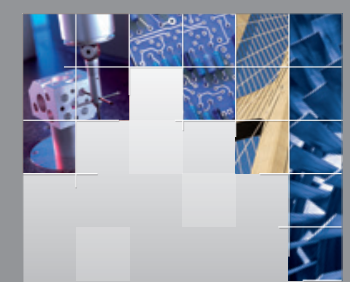

\section{Enfincering}
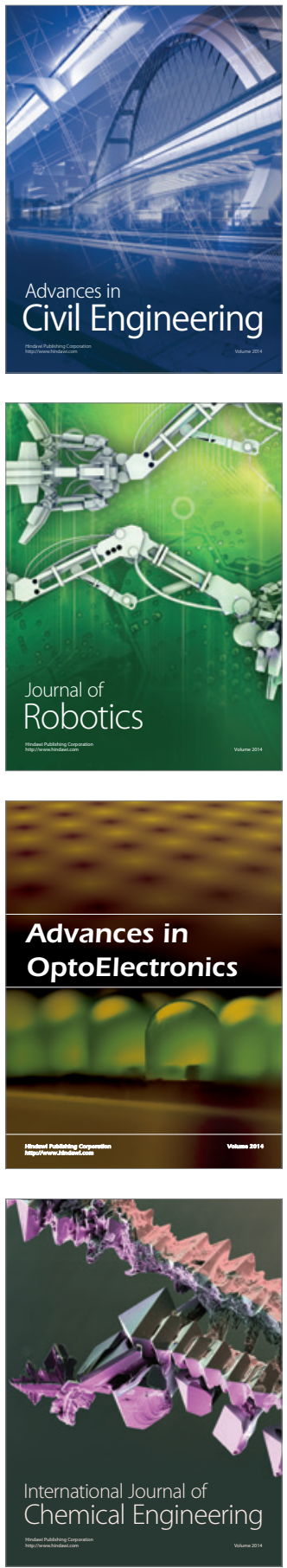

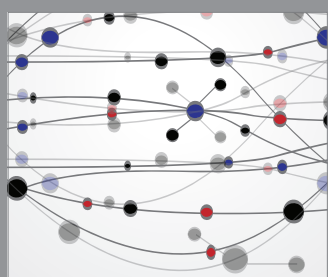

The Scientific World Journal

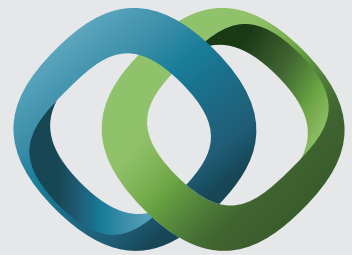

\section{Hindawi}

Submit your manuscripts at

http://www.hindawi.com
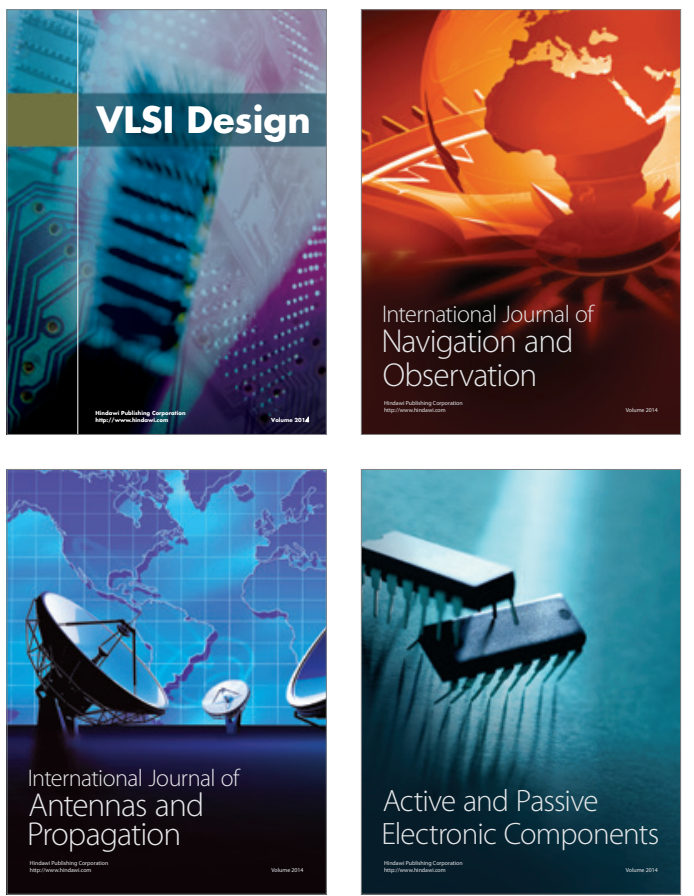
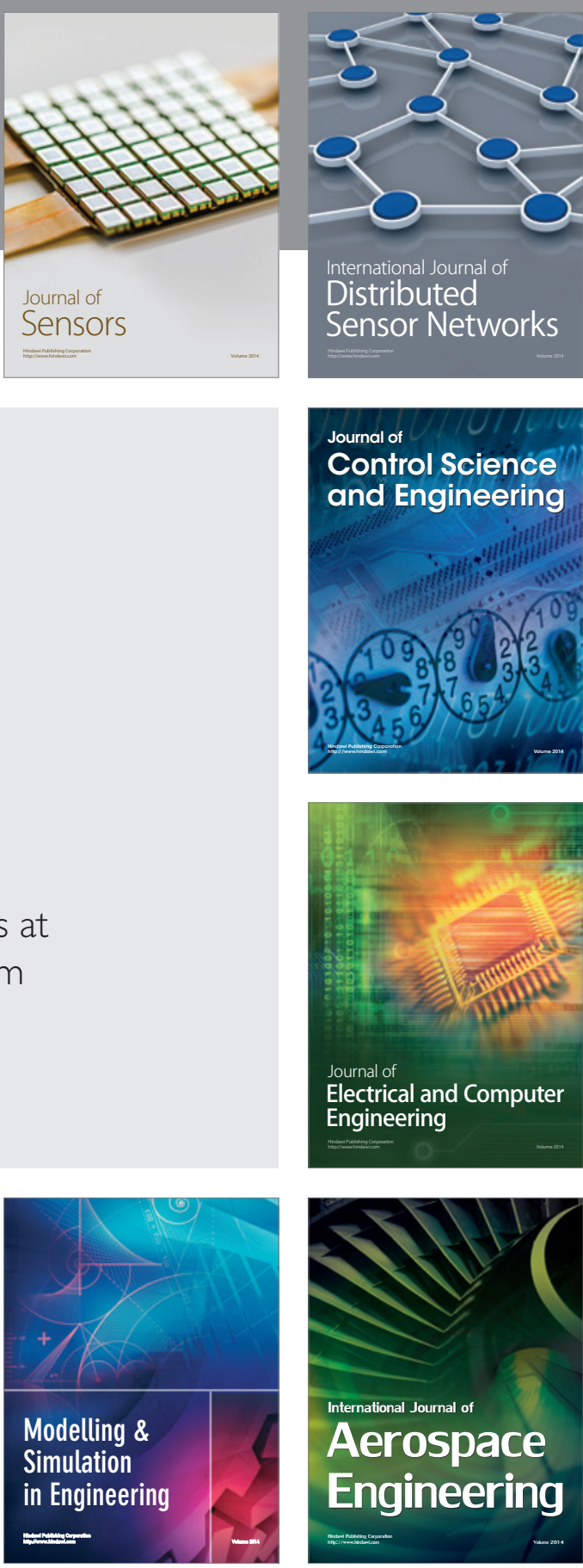

International Journal of

Distributed

Sensor Networks

Journal of

Control Science

and Engineering
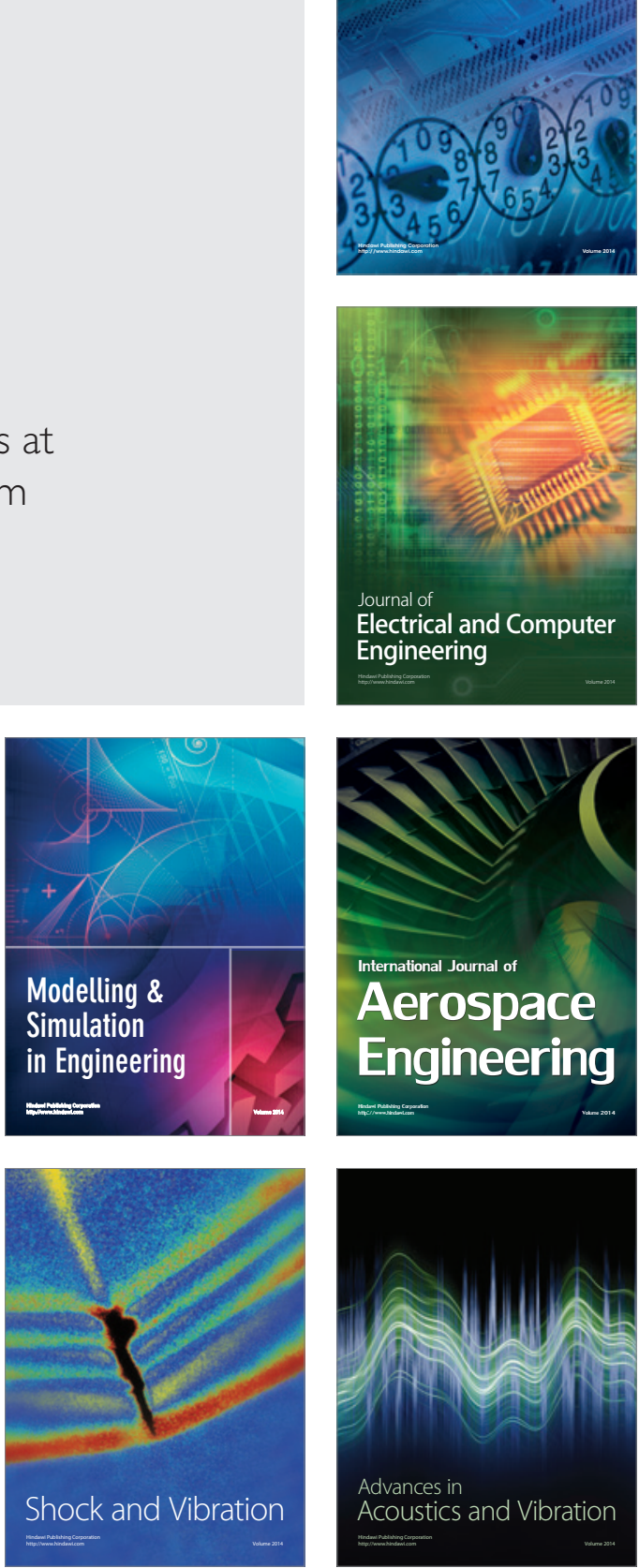